\title{
Effects of Neuromuscular Joint Facilitation on Electromechanical Reaction Time of Rectus Femoris
}

\author{
Ming Huo, PT, PhD ${ }^{1)}$, Meng Ge, PT, 2), Desheng Li, PT, $\mathrm{MS}^{3)}$, \\ QIUCHEN HUANG, PT $^{3)}$, HitOSHI MARUYAMA, PT, PhD ${ }^{1)}$ \\ 1) Department of Physical Therapy, Faculty of Health Science, International University of Health and \\ Welfare: 2600-1 Kitakanemaru, Ohtawara City, Tochigi 324-8501, Japan. \\ TEL+81287-24-3000,E-mail: huoming8@gmail.com \\ ${ }^{2)}$ Research Institute of Health and Welfare Sciences, Graduate School, International University of Health \\ and Welfare \\ ${ }^{3)}$ China Rehabilitation Research Center
}

\begin{abstract}
Purpose] The aim of this study was to investigate the change in the electromechanical (EMG) reaction time (RT) of the human rectus femoris after neuromuscular joint facilitation (NJF) treatment. [Subjects] The subjects were 17 healthy males who were divided into two groups: a NJF group and a control group. The NJF group consisted of 10 subjects, and the control group consisted of 7 subjects. [Methods] Participants in the NJF group received NJF treatment. We measured the EMG-RT, the premotor time (PMT) and the motor time (MT) during knee extension movement before, immediately after, and 10, 20 and 30 minutes after the intervention. [Results] There were no significant differences among the results of the control group. For the NJF group, there were significant differences in EMG-RT and PMT between pre- and post-intervention, and 10 and 30 minutes after exercise, and there was significant difference in MT between pre- and post-intervention. [Conclusion] NJF intervention shortens not only PMT but also MT, which indicates that NJF is effective for both premotor and motor processes.
\end{abstract}

Key words: Neuromuscular joint facilitation, Premotor time, Motor time

(This article was submitted Jul. 28, 2011, and was accepted Aug. 30, 2011)

\section{INTRODUCTION}

Neuromuscular Joint Facilitation (NJF) is a new therapeutic exercise based on kinesiology, that integrates the facilitation element of proprioceptive neuromusclar facilitation and the joint composition movement, aiming to improves the movement of the joint through passive exercise, active exercise and resistance exercise ${ }^{1)}$. It is used to increase strength, flexibility and ROM.

The electromechanical reaction time (EMG-RT) is an index of the shrinkage characteristic of the muscle. EMG$\mathrm{RT}$ is composed of premotor time (PMT) and motor time (MT). The PMT is defined as the interval between the stimulation signal and the onset of voluntary electromyographic (EMG) activity of a response agonist. The MT is defined as the interval between the onsets of voluntary EMG activity to the mechanical response ${ }^{2)}$. The PMT reflects the movement position, the movement pattern, the movement programming, the state of consciousness, the level of alertness and the time of central process. The MT chiefly reflects factors at the peripheral level, including muscle contraction, series elasticity, and difficulties in loosening of connective tissue and the joint capsule ${ }^{3)}$.

To evaluate muscle function, strength, reaction time and spatial coordinates are quantified ${ }^{4}$. The aim of this study was to investigate the change in the electromechanical reaction time (EMG-RT) of the human rectus femoris (RF) after neuromuscular joint facilitation (NJF) treatment.

\section{SUBJECTS AND METHODS}

The subjects were seventeen healthy males who were divided into two groups: a NJF group and a control group. The NJF group consisted of 10 subjects, and the control group consisted of 7 subjects. The detailed characteristics of the subjects are shown in Table 1. All subjects were screened before the onset of study by having them fill out a questionnaire. The questionnaire addressed whether the subjects had a history of cardiopulmonary, musculoskeletal, somatosensory or neurological disorders. If so, they were excluded from the study. All subjects gave their informed consent to participation in the study.

We measured the EMG-RT of the RF, the PMT of the $\mathrm{RF}$, and the MT of the RF in response to an auditory stimulus. The EMG-RT was measured with a digital storage oscilloscope DCS-7040 (Kenwood). After cleaning the skin with alcohol and abrasion paste, $\mathrm{Ag} / \mathrm{AgCl}$ disposable electrodes (Vitrode F, Nihonn Kohden) were placed over the muscle bellies of the RF with a $2-\mathrm{cm}$ inter-electrode distance. The signal was turned on and off by the contact of 
Table 1. Subject Characteristics

\begin{tabular}{lccr}
\hline & $\begin{array}{c}\text { NJF }^{\text {a }} \\
(\mathrm{n}=10)\end{array}$ & $\begin{array}{c}\text { Control }^{\mathrm{b}} \\
(\mathrm{n}=7)\end{array}$ & $\begin{array}{c}\text { Sum total } \\
(\mathrm{n}=17)\end{array}$ \\
\hline Age $(\mathrm{y})$ & $26.6 \pm 7.6$ & $28.7 \pm 7.8$ & $27.5 \pm 7.5$ \\
Height $(\mathrm{cm})$ & $173.8 \pm 8.7$ & $178.1 \pm 7.5$ & $175.6 \pm 8.2$ \\
Weight $(\mathrm{kg})$ & $70.0 \pm 8.4$ & $74.4 \pm 10.9$ & $71.8 \pm 9.5$ \\
\hline
\end{tabular}

Note: values are mean \pm standard deviation. There were no significant differences between the groups. a: NJF group: neuromuscular joint facilitation group. b: Control: control group.

an electrode attached to the calcaneus with an aluminum board. At the onset of voluntary knee extension, the electrode loses contact with the aluminum board, and the signal is turned off (Fig. 1).

The subjects sat on a backless, adjustable seat with the right leg fixed in a position of $90^{\circ}$ of hip flexion and $90^{\circ}$ of knee flexion. The subjects were given an oral warning of "Set" for 2 to 3 seconds in advance of the stimulus auditory signal $(2500 \mathrm{~Hz}, 50 \mathrm{~ms})$. The subjects were required to respond to the auditory cues by extending the knee as quickly as possible.

The EMG waveform and the on-off signal of the foot switch were synchronized on the display of the oscilloscope. The latent time between the onset of voluntary EMG activity and the stimulus auditory signal (PMT), and the latent time between the onset of voluntary EMG activity and the off signal (MT) were measured by setting the image on the display to a standstill each time and moving the cursor. Prior to the experiment, the subjects were informed of what would be done in the experiment and trial exercises were performed several times until subjects were accustomed to it. We measured EMG-RT, the premotor time (PMT) and the motor time (MT) during knee extension movement before, immediately after, and at 10, 20, and 30 minutes after the intervention in both groups. The reaction time was measured repeatedly for five times at each scheduled measurement.

Four knee patterns of NJF were used. The patterns were the knee extension-tibia external rotation (E-ER) pattern, the knee flexion-tibia internal rotation (F-IR) pattern, the knee extension-tibia internal rotation (E-IR) pattern, and the knee flexion-tibia external rotation (F-ER) pattern ${ }^{1)}$. Each pattern was performed three times at random as a passive exercise and as a resistance exercise. To measure the resistance force of the resistance exercise, two hand-held dynamometers (HHD, ANIMA MT-1) were held in each hand of a physical therapist, and the maximal resistance force was measured using the tester function of HHD. Table 2 shows the resistance force and duration of each procedure. In the NJF group intervention, both proximal resistance and distal resistance were performed. In the control group intervention, only distal resistance was performed. The intervention was done by the same physical therapist to avoid inter-rater errors.

To determine whether there were differences between the NJF group and the control group, the independent t-test was performed on subject characteristics. Two-way analysis of variance (ANOVA) was used to test for statistically

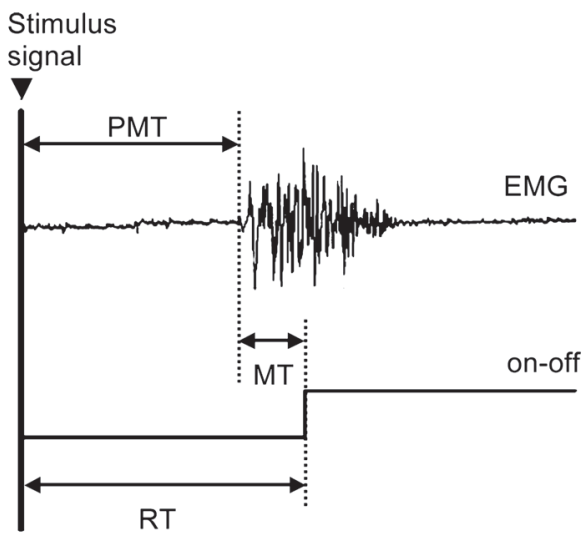

Fig. 1 Schema of the electromyographic reaction time

Upper trace: electromyographic waveform. Lower trace: on-off signal of the foot switch. The left vertical line shows the stimulus auditory signal. PMT: premotor time. MT: motor time. RT: electromechanical reaction time

Table 2. The resistance force and duration of NJF treatment for the Knee joint $(n=6)$

\begin{tabular}{lccc}
\hline Pattern & $\begin{array}{c}\text { Proximal resistance } \\
\text { force }(\mathrm{kg})\end{array}$ & $\begin{array}{c}\text { Distal resistance } \\
\text { force }(\mathrm{kg})\end{array}$ & $\begin{array}{c}\text { Duration of } \\
\text { procedure }(\mathrm{sec})\end{array}$ \\
\hline E-ER $^{\mathrm{a}}$ & $11.6 \pm 1.9$ & $14.3 \pm 2.1$ & $3.8 \pm 0.6$ \\
F-IR $^{\mathrm{b}}$ & $12.2 \pm 1.5$ & $11.2 \pm 1.9$ & $4.1 \pm 0.3$ \\
E-IR $^{\mathrm{c}}$ & $14.4 \pm 2.1$ & $9.9 \pm 0.8$ & $3.5 \pm 0.6$ \\
F-ER $^{\mathrm{d}}$ & $8.9 \pm 1.1$ & $12.2 \pm 1.8$ & $4.2 \pm 0.4$ \\
\hline
\end{tabular}

a: E-ER: knee extension-tibia external rotation pattern. ${ }^{\mathrm{b}}$ : F-IR: knee flexion-tibia internal rotation pattern. ${ }^{c}$ : E-IR: knee extension- tibia internal rotation pattern. ${ }^{\mathrm{d}}$ : F-ER: knee flexion- tibia external rotation pattern.

significant differences, and the factors were intervention and group. If a significant interaction was found, one-way ANOVA and multiple comparisons (Bonferroni test) were performed for each group. Data were analyzed using SPSS Ver. 12.0 for Windows. The level of statistical significant was chosen as 0.05 .

\section{RESULTS}

There were no significant differences in the subject characteristics between the NJF group and the control group.

Table 3 shows the results for reaction times. In two-way analysis of variance, there was a significant interaction of group, indicating that the change in RT was different between the groups. There were no significant differences among the results of the control group. In the NJF group, there were significant differences in EMG-RT and PMT between pre- and post-intervention, and 10 and 30 minutes after exercise, and there was a significant difference in MT between pre- and post-intervention. 
Table 3. Comparison before and after intervention of each measurement item (msec)

\begin{tabular}{llccccc}
\hline & & before & just after exercise & after 10 min & after 20 min & after 30 min \\
\hline \multirow{3}{*}{ NJF a } & PMT $^{\mathrm{c}}$ & $124.7 \pm 24.4$ & $107.3 \pm 21.5^{* *}$ & $104.1 \pm 18.6^{*}$ & $105.1 \pm 16.2^{*}$ & $106.2 \pm 17.0^{*}$ \\
& MT $^{\mathrm{d}}$ & $44.4 \pm 7.6$ & $35.6 \pm 5.1^{* *}$ & $41.4 \pm 6.0$ & $39.7 \pm 8.7$ & $39.0 \pm 7.8$ \\
& RT $^{\mathrm{e}}$ & $197.1 \pm 25.7$ & $170.0 \pm 20.6^{* *}$ & $174.8 \pm 18.9^{*}$ & $171.0 \pm 16.2^{* *}$ & $170.6 \pm 14.8^{* *}$ \\
\hline \multirow{3}{*}{ Control b b } & PMT & $140.8 \pm 17.3$ & $135.3 \pm 12.8$ & $139.9 \pm 12.8$ & $136.9 \pm 12.0$ & $133.3 \pm 9.7$ \\
& MT & $46.3 \pm 7.9$ & $46.2 \pm 10.1$ & $44.2 \pm 9.4$ & $45.9 \pm 8.7$ & $44.3 \pm 9.4$ \\
& RT & $187.1 \pm 17.4$ & $181.5 \pm 16.0$ & $184.1 \pm 13.3$ & $182.8 \pm 12.9$ & $177.5 \pm 10.3$ \\
\hline
\end{tabular}

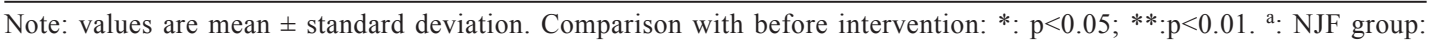
neuromuscular joint facilitation group. ${ }^{b}$ : Control: control group. ${ }^{c}:$ PMT: premotor time. ${ }^{d}:$ MT: motor time. ${ }^{e}:$ RT: electromechanical reaction time; RT= PMT + MT.

\section{DISCUSSION}

This study investigated the effects of a Neuromuscular Joint Facilitation treatment on EMG-RT. The NJF group subjects showed not only shortened PMT, but also shortened MT in the rectus femoris. The shortened PMT means that arousal levels and attentiveness were improved, and the reaction time became faster as a result of the NJF intervention. In addition, MT was shortened immediately after intervention, and a possible reason for this is that the contractile characteristic of the muscle was changed by the NJF intervention. MT is influenced by muscle tone before the movement appears ${ }^{5}$. The shortened MT is presumed to have been caused by an increase in muscular tension which was induced by change in mechanical properties of the tissue $^{6)}$. The shortened RT is presumed to have been the result of the shortening of both PMT and MT immediately after the intervention, and subsequently by the shortening PMT at 30 minutes.

There was no significant difference among the results of the control group. This was in agreement with the results of a previous study, which concluded that resistance training did not change RT (premotor time, motor time, or reaction

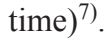

NJF intervention shortens not only PMT but also MT, which indicates that NJF is effective for both premotor and motor processes.

\section{REFERENCES}

1) Huo M: Neuromuscular Joint Facilitation. Tokyo: Ipec press, 2010, pp3, 127-138 (in Japanese)

2) Nakamura R: Clinical Kinesiology. Tokyo: Ishiyaku press, 2002, pp270 (in Japanese).

3) Nakamura R: Examination of movement appearance by electromyogram response time. Rinsyounouha, 1988, 30: 566-572(in Japanese).

4) Inohara $\mathrm{H}$, Katou H, Kitou N: Conservative Therapy of Osteoarthrosis seen from Multijoint Kinetic Chain. Tokyo: Zennihonbyouin press, 2008, pp35 (in Japanese)

5) Ward T: Muscle state: Reaction and Movement time in elbow extension. Arch Phys Med Rehab, 1978, 59: 377-383.

6) Schmidt RA, Stull GA: Premotor and motor reaction time as a function of preliminary muscular tention. J Motor Behav, 1970, 2: 96-110.

7) Anonymous: Sport medicine; new sports medicine findings from D. P. Laroche and co-authors described. Medical Sciences, 2008, 2054. 